\title{
ON $\alpha$-HARMONIC FUNCTIONS
}

\author{
MASAYUKI ITÔ
}

\section{Chapter 1. Introduction and Preliminaries}

M. Riesz [8] introduced the notion of $\alpha$-superharmonic functions in $n(\geq 1)$. dimensional Euclidean space $R^{n}$ in connection with the potential of order $\alpha$. In this paper, we shall first define the $\alpha$-superharmonic and $\alpha$-harmonic functions in a domain $D$. In case $\alpha=2$, they coincide with ones in the usual sense. Next we shall introduce generalized Laplacians $\underline{P}_{f}^{\alpha}(x)$ and $P_{f}^{\alpha}(x)$ of order $\alpha$, which are, in the case $\alpha=2$, equal to the well-known generalized Laplacians except for a universal constant. Then we shall prove the following equivalences.

1. A Lebesgue measurable function $f(\neq+\infty)$ in $R^{n}$ is $\alpha$-superharmonic in a domain $D$ if and only if $f$ is lower semicontinuous and $\underline{P}_{f}^{\alpha}(x) \leq 0$ in $D$.

2. A Lebesgue measurable function $f$ in $R^{n}$ is $\alpha$-harmonic in a domain $D$ if and only if $f$ is finite continuous in $D$ and $P_{f}^{\alpha}(x)=0$ in $D$.

Finally we shall prove Ninomiya's domination principle as an application of the above results.

In $R^{n}$, the potential of a given order $\alpha, 0 \leq \alpha<n$, of a measure $\mu$ in $R^{n}$ is defined by

$$
U_{\alpha}^{\mu}(x)=\int|x-y|^{\alpha-n} d \mu(y)
$$

provided the integral on the right exists. We shall say that a measure $\mu$ in $R^{n}$ is $\alpha$-finite if the potential $U_{\alpha}^{\mu}(x)$ is finite p.p.p. in $R^{n}$. Here a property is said to hold p.p.p. on a subset $X$ in $R^{n}$, when the property holds on $X$ except for a set $E$ which does not support any measure $\nu \neq 0$ with finite $\alpha$-energy $\iint|x-y|^{\alpha-n} d \nu(y) d_{\nu}(x)$. M. Riesz [8] proved that every $\alpha$-finite measure can be balayaged to every closed set if $0<\alpha \leq 2,0<\alpha<2$ or $0<\alpha<1$ according to $n \geq 3, n=2$ or $n=1$. This paper is based on this result. Let $F$ be a closed set in $R^{n}$ and $x$ be a point in $\mathscr{C} F$. We shall denote the balayaged measure

Received August 31, $196 j$. 
of a unit measure $\varepsilon_{x}$ at $x$ to $F$ by $\mu_{x, F}^{(\alpha)}$. Let $B\left(x_{0} ; r\right)$ be an open ball with center $x_{0}$ and radius $r$. If $\alpha \neq 2$, for any $x$ in $B\left(x_{0} ; r\right)$,

$$
d \mu_{x, \mathscr{C} B\left(x_{0} ; r\right)}^{(\alpha)}(y)=\lambda_{x_{0, r}}(x, y) d y
$$

with

$$
\lambda_{x_{0}, r}(x, y)=\left\{\begin{array}{l}
a_{\alpha}\left(r^{2}-\left|x-x_{0}\right|^{2}\right)^{\alpha / 2}\left(\left|y-x_{0}\right|^{2}-r^{2}\right)^{-\alpha / 2}|y-x|^{-n} \text { in } \mathscr{C} B\left(x_{0} ; r\right) \\
0 \text { in } B\left(x_{0} ; r\right),
\end{array}\right.
$$

where

$$
a_{\alpha}=\pi^{-(\alpha / 2+1)} \Gamma\left(\frac{n}{2}\right) \sin \frac{\alpha n}{2}
$$

It holds that

$$
\int d \mu_{x, F}^{(\alpha)} \leq 1 \text { and } \int \kappa_{x_{0}, r}(y) d y=1
$$

where

$\kappa_{x_{0}, r}(y)$ stands for $\lambda_{x_{0}, r}\left(x_{0}, y\right)$. For a given real-valued function $f$ Lebesgue measurable in $R^{n}$, we shall denote

$$
\int f(y)_{\kappa_{x_{0}}, r}(y) d y
$$

by $\mathfrak{M}_{\alpha}\left(x_{0} ; f, r\right)$. This is a generalization of Gauss' mean value.

\section{Chapter 2. $\alpha$-harmonic functions}

Throughout this chapter, we assume that $0<\alpha<2$ or $0<\alpha<1$ according to $n \geq 2$ or $n=1$. A measure with density $f$, measurable in $R^{n}$, will be called the measure $f$. First we shall define $\alpha$-superharmonic functions and $\alpha$-harmonic functions.

\section{§ 2.1. Definitions}

Definition 1.3) Let $D$ be a domain in $R^{n}$. We shall say that a function $f$ defined in $R^{n}$ is $\alpha$-superharmonic in $D$ if $f$ satisfies the following three conditions :

1) The notion of $\alpha$-superharmonicity was first introduced by M. Riesz [8]. According to him, a function $f$ is $\alpha$-superharmonic in $R^{n}$ if $f$ satisfies the following conditions:

(1) $f(x) \geq 0$ and $f(x)$ 丰 $+\infty$ in $R^{n}$,

(2) $f$ is lower semicontinuous in $R^{n}$,

(3) for each $x$ in $R^{n}$ and each open ball $B(x ; r), f(x) \geq \mathbb{M}_{\alpha}(x ; f, r)$. Another kind of $\alpha$-superharmonicity was introduced by Frostman [4]. 
(S. 1) $f$ is Lebesgue measurable in $R^{n}$,

(S. 2) $f$ is lower semicontinuous in $D$,

(S. 3) for each $x$ in $D$ and each open ball $B(x ; r)$ contained with its closure in $D, \mathfrak{R}_{\alpha}(x ; f, r)$ exists and

$$
f(x) \geq \mathfrak{M}_{\alpha}(x ; f, r) .
$$

DEFinition 2. Let $D$ be a domain in $R^{n}$. We shall say that a function $f$ defined in $R^{n}$ is $\alpha$-harmonic in $D$ if $f$ satisfies the following three conditions:

(H. 1) $f$ is Lebesgue measurable in $R^{n}$,

(H. 2) $f$ is finite continuous in $D$,

(H. 3) for each $x$ in $D$ and each open ball $B(x ; r)$ contained with its closure in $D, \mathfrak{M}_{\alpha}(x ; f, r)$ exists and

$$
f(x)=\mathfrak{M}_{a}(x ; f, r) .
$$

It is easily seen that the potential $U_{\alpha}^{\mu}(x)$ of an $\alpha$-finite positive measure $\mu$ is $\alpha$-superharmonic in $R^{n}$ and $\alpha$-harmonic in $\mathscr{C} S_{\mu}{ }^{21}$

\section{§ 2. 2. Elementary properties}

Property 1. Let $f$ and $f^{\prime}$ be $\alpha$-harmonic in a domain D. If $f(x)=f^{\prime}(x)$ in $D$, then $f(x)=f^{\prime}(x)$ almost everywhere in $R^{n}$. In fact, for any open ball $B\left(x_{0}\right.$; $\left.r_{0}\right)$ contained with its closure in $D$ and any $x$ in $B\left(x_{0} ; r_{0}\right)$, it holds that

$$
\begin{aligned}
& \int\left(f(y)-f^{\prime}(y)\right) \lambda_{x_{0}, r_{0}}(x, y) d y \\
= & a_{\alpha}\left(r_{0}^{2}-\left|x-x_{0}\right|^{2}\right)^{\alpha / 2} \int_{\mathscr{B} B\left(x_{0} ; r_{0}\right)}\left(f(y)-f^{\prime}(y)\right)\left(\left|y-x_{0}\right|^{2}-r_{\theta}^{2}\right)^{-\alpha / 2}|y-x|^{-n} d y \\
= & f(x)-f^{\prime}(x)=0
\end{aligned}
$$

by Lemma 4 which we shall be given in $\S 2.3$. Put

$$
g(x)=\left\{\begin{array}{l}
0 \text { in } B\left(x_{0} ; r_{0}\right) \\
\left(f(x)-f^{\prime}(x)\right)\left(\left|x-x_{0}\right|^{2}-r_{0}^{2}\right)^{-\alpha / 2} \text { on } C B\left(x_{0} ; r_{0}\right) .
\end{array}\right.
$$

Then the potential of order 0 of the measure $g$ is équal to 0 in $B\left(x_{0} ; r_{0}\right)$. By the unicity theorem of M. Riesz ${ }^{3)}, g(x)=0$ almost everywhere in $R^{n}$. Hence $f(x)=f^{\prime}(x)$ almost everywhere in $\mathscr{C} B\left(x_{0} ; r_{0}\right)$. This completes the proof.

2) $\mathrm{Cf}$, [8], $n^{\circ} 20$.

3) Cf. [8], $n^{\circ} 11$. 
Property 2. If $f$ is harmonic in the usual sense in $R^{n}$, it is $\alpha$-harmonic there. In fact, let $x_{0}$ be a point in $R^{n}$ and $r$ be a positive number. Using the polar coordinate $(\rho, \sigma)$ with center at $x_{0}$, we have

$$
M_{\alpha}\left(x_{0} ; f, r\right)=a_{\alpha} r^{\alpha} \int_{r}^{\infty}\left(\rho^{2}-r^{2}\right)^{-\alpha / 2 \rho-1}\left(\int_{s\left(x_{0} ; 1\right)} f_{\rho_{0},} d \sigma\right) d \rho,
$$

where $S\left(x_{0} ; 1\right)$ is a unit sphere with center $x_{n}$. Since $f$ is harmonic in the usual sense in $R^{n}$,

$$
f\left(x_{0}\right)=\frac{1}{\omega_{n}} \int_{s\left(x_{0} ; 1\right)} f_{p, s} d \sigma,
$$

where $\omega_{n}$ denotes the area of the unit sphere. Hence

$$
f\left(x_{0}\right)=\mathfrak{M}_{\alpha}\left(x_{0} ; f, r\right) .
$$

Property 3. If $f$ is $\alpha$-harmonic and bounded from below in $R^{n}$, then it is constant. In fact, without loss of generality we may assume that $f$ is nonnegative. By M. Riesz's decomposition theorem ${ }^{4}$, there exist $\alpha$-finite positive measure $\nu$ and a non-negative constant $C$ such that

$$
f(x)=U_{\alpha}^{\nu}(x)+C
$$

in $R^{n}$. Suppose that $f$ is non-constant. Then there exist a point $x_{0}$ in $R^{n}$ and a positive number $r_{0}$ such that $\nu\left(B\left(x_{0} ; r_{0}\right)\right)>0$. Let $\nu^{\prime}$ be the balayaged measure of $\nu$ to $\mathscr{C} B\left(x_{0} ; r_{0}\right)$. For any $x$ in $B\left(x_{0} ; r_{0}\right)$,

$$
\begin{aligned}
& U_{\alpha}^{\nu^{\prime}}\left(x_{0}\right)=\int U_{\alpha}^{\nu}(y) \lambda_{x_{0}, r_{0}}(x, y) d y=\int|y-z|^{\alpha-n} \lambda_{x_{0}, r_{0}}(x, y) d y d \nu(z) \\
< & \int U_{\alpha}^{\varepsilon_{x}}(y) d \nu(y)=U_{x}^{\nu}(x) .
\end{aligned}
$$

In particular,

$$
U_{\alpha}^{\nu}\left(x_{0}\right)>\int U_{\alpha}^{\nu}(y) \kappa_{x_{0}, r_{0}}(y) d y=\mathfrak{M}_{\alpha}\left(x_{0} ; U_{\alpha}^{\nu}, r_{0}\right)
$$

This contradicts our assumptions.

Property 4. Let $f$ be harmonic in the usual sense in $R^{n}$. If it is bounded from below, it is constant. This follows from Properties 2 and 3 .

PROPERTy 5. Let $f$ be $\alpha$-harmonic in $R^{n}$. If there exist an $\alpha$-finite positive

1) Cf. [8], $n^{\circ} 31$ and $n^{\circ} 32$. 
measure $\nu$ and a positive constant $C$ such that

$$
|f(x)| \leq U_{\alpha}^{\nu}(x)+C
$$

in $R^{n}$, then $f$ is constant. In fact, for any $x_{0}$ in $R^{n}$ and any positive number $r$,

$$
\begin{aligned}
& \left|f\left(x_{0}\right)\right|=\left|\int_{\mathscr{C} B\left(x_{0} ; r\right)} f(y) \kappa_{x_{0}, r}(y) d y\right| \leq \int_{\mathscr{C}_{\left.B i x_{0} ; r\right)}}|f(y)| \kappa_{x_{0}, r}(y) d y \\
& \leq \int_{\mathscr{C} B\left(x_{0}: r\right)}\left(U_{\alpha}^{\nu}(y)+C\right) \kappa_{x_{0}, r}(y) d y=\mathfrak{M}_{\alpha}\left(x_{0} ; U_{\alpha}^{\nu}, r\right)+C .
\end{aligned}
$$

Since $\lim _{r \rightarrow \infty} \mathfrak{M}_{\alpha}\left(x_{0} ; U_{\alpha}^{\nu}, r\right)=0^{5)},\left|f\left(x_{0}\right)\right| \leq C$.

By Property $3, f$ is constant.

Property 6. Let $f$ be harmonic in the usual sense in $R^{n}$. If there exist an $\alpha$-finite positive measure " and a non-negative constant $C$ such that

$$
|f(x)| \leq U_{\alpha}^{\nu}(x)+C
$$

in $R^{n}$, then $f$ is constant. This follows from Properties 2 and 5.

\section{§ 2.3. Four Lemmas}

Let $D$ be a domain in $R^{n}$ and a function $f$ defined in $R^{n}$ be $\mu_{x, \mathscr{C}}^{(\alpha)}$-integrable for any $x$ in $D$. We denote by $E_{f, n}(x)$ the following function

$$
\left\{\begin{array}{l}
f(x) \text { in } \mathscr{C} D \\
\int f(y) d \mu_{x, \mathscr{C} D}^{(\alpha)}(y) \text { in } D .
\end{array}\right.
$$

LEMMA 1. Let $B\left(x_{0} ; r_{0}\right)$ be an open ball and $f$ be a Lebesgue measurable and bounded function in $R^{n}$. Then $E_{f, B\left(x_{0} ; r_{0}\right)}(x)$ is $\alpha$-harmonic in $B\left(x_{0} ; r_{0}\right)$.

Proof. Evidently $E_{f, B\left(x_{0} ; r_{0}\right)}(x)$ is finite continuous in $B\left(x_{0} ; r_{0}\right)$. Hence it is sufficient to prove the condition (H.2). By Lusin's theorem, there exists a sequence $\left(f_{m}\right)$ of functions of class $C^{2}$ with compact support such that $f_{m}(x)$ $\rightarrow f(x)$ almost everywhere in $R^{n}$ as $m \rightarrow \infty$, and

$$
\left|f_{m}(x)\right| \leq M,|f(x)| \leq M \text { in } R^{n}
$$

where $M$ is a positive constant. Since $f_{m}$ is of class $C^{2}$ with compact support,

$$
f_{m}(x)=\int|x-y|^{\alpha-n_{m}} k_{m}(y) d y
$$

where

5) Cf. [8], $n^{\circ} 31$, 


$$
k_{n}(y)=\int|y-z|^{\left(2-\alpha_{i}-n\right.} \Delta f_{m}(z) d z
$$

Let $\mu_{m}$ be the balayaged measure of the measure $k_{m}$ to $\mathscr{C} B\left(x_{0} ; r_{0}\right)$. Then

$$
U_{\alpha}^{\mu_{m}}(x)=\left\{\begin{array}{l}
f_{m}(x) \text { on } \mathscr{C} B\left(x_{0} ; r_{0}\right) \\
\int f_{m}(y) \lambda_{x_{0}, r_{0}}(x, y) d y \text { in } B\left(x_{0} ; r_{0}\right) .
\end{array}\right.
$$

By Lebesgue's bounded convergence theorem,

$$
U_{\alpha}^{\mu_{m}}(x) \rightarrow E_{f, B\left(x_{0} ; r_{0}\right)}(x)
$$

almost everywhere in $R^{n}$ as $m \rightarrow \infty$. On the other hand, being

$$
\int \lambda x_{x_{0}, r_{0}}(x, y) d y \leq 1
$$

it holds that

$$
\left|U_{\alpha}^{\mu_{m}}(x)\right| \leq M \text { in } R^{n} .
$$

Hence by Lebesgue's bounded convergence theorem,

$$
\int U_{\alpha}^{\mu_{m}}(y) \kappa_{x_{1}, r}(y) d y \rightarrow \int E_{f_{,}\left(x_{0} ; r_{0}\right)}(y) \kappa_{x_{1}, r}(y) d y
$$

as $m \rightarrow \infty$ for any open ball $B\left(x_{1} ; r\right)$ contained with its closure in $B\left(x_{0} ; r_{0}\right)$. Since $S_{\mu_{m}} \subset \mathscr{C} B\left(x_{0} ; r_{0}\right) \subset \mathscr{C} B\left(x_{1} ; r\right)$,

$$
U_{\alpha}^{\mu m}\left(x_{1}\right)=\int U_{\alpha}^{\mu_{m}}(y)_{\kappa_{1}, r}(y) d y .
$$

Consequently

$$
E_{f, B\left(x_{0} ; r_{0}\right)}\left(x_{1}\right)=\mathfrak{M}_{\alpha}\left(x_{1}, E_{\left.f, B\left(x_{0} ; r_{0}\right), x\right)}\right.
$$

This completes the proof.

Lemma 2. Let $B\left(x_{0} ; r_{0}\right)$ be an open ball and a function $f$ be Lebesgue

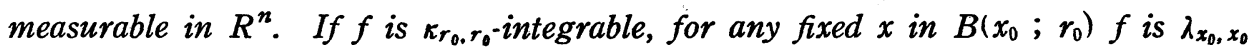
$(x, y)$-integrable and $E_{f, B\left(x_{0}: r_{0}\right)}(x)$ is a-harmonic in $B\left(x_{0} ; r_{0}\right)$.

Proof. First we shall show that in $B\left(x_{0} ; r_{0}\right)$

$$
\int|f(y)| \lambda_{r_{0} \cdot r_{0}}(x, y) d y<+\infty \text {. }
$$

In fact, for any fixed $x$ in $B\left(x_{0} ; r_{0}\right)$, there exists a positive constant $M$ such that 


$$
|y-x|^{-n} \leq M\left|y-x_{0}\right|^{-n}
$$

for any $y$ in $\mathscr{C} B\left(x_{0} ; r_{0}\right)$. Now

$$
\begin{aligned}
& \int|f(y)| \lambda_{x_{0}, r_{0}}(x, y) d y \\
= & a_{\alpha}\left(r_{0}^{2}-\left|x-x_{0}\right|^{2}\right)^{\alpha / 2} \int_{\mathscr{C} B\left(x_{0} ; r_{0}\right)}|f(y)|\left(\left|y-x_{0}\right|^{2}-r_{0}^{2}\right)^{-\alpha / 2}|y-x|^{-n} d y \\
\leq & M\left(r_{0}^{2}-\left|x-x_{0}\right|^{2}\right)^{\alpha / 2} r_{0}^{-\alpha} \int|f(y)| \kappa_{x_{0}, r_{0}}(y) d y<+\infty .
\end{aligned}
$$

Similarly as Lemma $1, E_{f, B\left(x_{0} ; r_{0}\right)}(x)$ is finite continuous in $B\left(x_{0} ; r_{0}\right)$. Put

$$
f_{m}^{+}(x)=\inf \left(f^{+}(x), m\right), f_{m}^{-}(x)=\inf \left(f^{-}(x), m\right),
$$

where

$$
f^{+}(x)=\sup (f(x), 0), f^{-}(x)=-\inf (f(x), 0) .
$$

By Lemma $1, E_{f_{m}, B\left(x_{0} ; r_{0} !\right.}(x)$ and $E_{f_{m}, B\left(x_{0} ; r_{0}\right)}(x)$ are $\alpha$-harmonic in $B\left(x_{0} ; r_{0}\right)$. Hence

$$
E_{f_{m}, B\left(x_{0} ; r_{0}\right)}(x)=\mathfrak{M}_{\alpha}\left(x ; E_{f_{m}, B\left(x_{0} ; r_{0}\right)}, r\right),
$$

and

$$
E_{f_{m}^{-}, B\left(x_{0} ; r_{0}\right)}(x)=\mathfrak{M}_{\alpha}\left(x ; E_{f_{m}^{-}, B\left(x_{0} ; r_{0}\right)}, r\right),
$$

for any open ball $B(x ; r)$ contained with its closure in $B(x ; r)$. Since $\left(E_{f_{m}^{+}, B\left(x_{0} ; r_{0}\right)}\right)$ tends increasingly to $E_{f+, B\left(x_{0} ; r_{0}\right)}$,

$$
\mathfrak{R}_{\alpha}\left(x ; E_{f_{m}, B\left(x_{0} ; r_{0}\right)}^{+}, r\right) \rightarrow \mathfrak{M}_{\alpha}\left(x ; E_{f^{+}, B\left(x_{0} ; r_{0}\right), r}\right)
$$

as $m \rightarrow \infty$. Consequently

$$
E_{f^{+}, B\left(x_{0} ; r_{0}\right)}(x)=\mathfrak{M}_{\alpha}\left(x ; E_{f^{+}, B\left(x_{0} ; r_{0}\right), r}\right)
$$

for any $x$ in $B\left(x_{0} ; r_{0}\right)$ and any open ball $B(x ; r)$ contained with its closure in $B\left(x_{0} ; r_{0}\right)$. Similarly we obtain that

$$
E_{f-, B\left(x_{0} ; r_{0}\right)}(x)=\mathfrak{D}_{\alpha}\left(x ; E_{f-, B\left(x_{0} ; r_{0}\right)}, r\right) .
$$

Therefore

$$
\begin{aligned}
& E_{f, B\left(x_{0} ; r_{0}\right)}(x)=E_{f^{+}, B\left(x_{0} ; r_{0}\right)}(x)-E_{f-, B\left(x_{0} ; r_{0}\right)}(x) \\
= & \mathfrak{N}_{\alpha}\left(x ; E_{f^{+}, B\left(x_{0} ; r_{0}\right)}, r\right)-\mathfrak{M}_{\alpha}\left(x ; E_{f^{-}, B\left(x_{0} ; r_{0}\right)}, r\right) \\
= & \mathfrak{M}_{\alpha}\left(x ; E_{f, B\left(x_{0} ; r_{0}\right)}, r\right) .
\end{aligned}
$$

This completes the proof. 
For a general domain $D$, we get in the same way the following

Lemma $2^{\prime}$. Let $D$ be a domain in $R^{n}$ and a function $f$ be Borel measurable

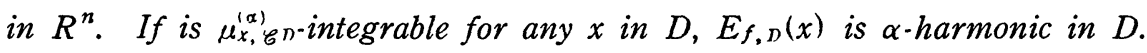

Lemma 3. Let a function $f$ be $\alpha$-harmonic in a bounded domain $D$. If $f$ is finite continuouus on $\bar{D}$ and $f(x)=0$ almost everywhere in $\mathscr{C} D$, then $f(x)=0$ in D.

Proof. Let $x_{0}$ be a point in $\bar{D}$ such that

$$
f\left(x_{0}\right)=\max \{f(x) ; x \in \bar{D}\} .
$$

Suppose that $f\left(x_{0}\right)>0$. Then $x_{0}$ is not on the boundary of $D$. Let $B\left(x_{0} ; r\right)$ be an open ball contained with its closure in $D$. Then

$$
\begin{aligned}
& \mathfrak{M}_{\alpha}\left(x_{0} ; f, r\right)=\int f(y) \kappa_{x_{0}, r}(y) d y \\
= & \int_{\mathscr{C} B\left(x_{i} ; r\right) \cap D} f(y) \kappa_{x_{0}, r}(y) d y \leq \int_{\mathscr{C} B\left(x_{0} ; r\right) \cap D} f\left(x_{0}\right) \kappa_{x_{0}, r}(y) d y \\
< & \int f\left(x_{0}\right) \kappa_{x_{0}, r}(y) d y=f\left(x_{0}\right) .
\end{aligned}
$$

This contradicts the $\alpha$-harmonicity of $f$. Therefore $f(x) \leq 0$ in $D$. Similarly we obtain $f(x) \geq 0$ in $D$, and hence $f(x)=0$ in $D$.

Lemma 4. Let $f$ be a-harmonic in a domain D. For each open ball contained with its closure in $D$,

$$
f(x)=\int f(y) \lambda_{x_{0}, r}(x, y) d y
$$

in $B\left(x_{0} ; r\right)$ and $f$ is analytic in $D$.

Proof. Similarly as Lemma 2, for any $x$ in $B\left(x_{0} ; r\right)$,

$$
\int|f(y)| \lambda_{x_{0}, r}(x, y) d y<+\infty .
$$

By Lemma $2, E_{f, r\left(x_{0} ; r\right)}(x)$ is $\alpha$-harmonic in $B\left(x_{0} ; r\right)$. Put

$$
g(x)=f(x)-E_{f, B\left(x_{0} ; r\right)}(x) .
$$

Then $g(x)=0$ in $B\left(x_{0} ; r\right)$. Consequently in $B\left(x_{0} ; r\right)$,

$$
f(x)=\int f(y) \lambda_{x_{0}} r(x, y) d y \text {. }
$$


Hence by M. Riesz's theorem ${ }^{6}, f$ is analytic in $B\left(x_{0} ; r\right) . \quad B\left(x_{0} ; r\right)$ being arbitrary, $f$ is analytic in $D$. This completes the proof.

\section{§2.4. Extension of generalized Laplacian}

Now we shall introduce another mean value of a function. Let $f$ be a Lebesgue measurable function in $R^{n}$. If

$$
\gamma \int_{1}^{\infty} \rho^{-\gamma-1}\left(\rho^{2}-1\right)^{r / 2-1} \cdot M \lambda_{\alpha}(x ; f, r \rho) d \rho
$$

exists for a positive nember $r$, we denote it by $\mathscr{A}_{\alpha, \gamma}(x ; f, r)$. Since

$$
r \int_{1}^{\infty} \rho^{-r-1}\left(\rho^{2}-1\right)^{\gamma / 2-1} d \rho=1
$$

$\mathscr{A}_{\alpha, \gamma}(x ; f, r)$ is considered as a kind of mean values of $f$. By M. Riesz's formula,

$$
\begin{aligned}
\mathscr{A}_{\alpha, r}(x ; f, r) \\
=C_{\alpha, r, n} r^{\alpha} \int_{\mathscr{C} B(x ; r)}\left(|x-y|^{2}-r^{2}\right)^{\gamma / 2-\alpha / 2}|x-y|^{-\gamma-n} f(y) d y,
\end{aligned}
$$

where

$$
C_{\alpha, \Upsilon, n}=\frac{\pi^{-n / 2} \Gamma\left(\frac{n}{2}\right) \Gamma\left(1+\frac{\gamma}{2}\right)}{\Gamma\left(\frac{\alpha}{2}\right) \Gamma\left(1+\frac{\alpha}{2}\right)}
$$

We denote the mean value corresponding to $\gamma=\alpha$ by $\mathscr{A}_{\alpha}(x ; f, r)$. Thus

$$
\mathscr{A}_{\alpha}(x ; f, r)=\frac{\alpha r^{\alpha}}{\omega_{n}} \int_{\mathscr{C} B(x ; r)}|x-y|^{-\alpha-n} f(y) d y
$$

We denote

$$
\lim _{\varepsilon \rightarrow 0} \frac{\omega_{n}}{\alpha \varepsilon^{\alpha}}\left(\mathscr{A}_{\alpha}(x ; f, \varepsilon)-f(x)\right)
$$

by $\underline{P}_{f}^{\alpha}(x)$. In particular, when

$$
\lim _{\varepsilon \rightarrow 0} \frac{\omega_{n}}{\alpha \varepsilon^{\alpha}}\left(\mathscr{A}_{\alpha}(x ; f, \varepsilon)-f(x)\right)
$$

exists, we denote it by $P_{f}^{\alpha}(x)$. For $\alpha=2, P_{f}^{\alpha}(x)$ coincides with the generalized Laplacian except for a universal constant ${ }^{71}$.

6) Cf. [8], $n^{\circ} 26$.

7) Cf. [1], pp. 17-18, 


\section{§ 2.5. Inverse distribution of $\boldsymbol{r}^{\alpha-n}$}

We consider the distribution $D_{\alpha}$ such that

$$
D_{\alpha} * r^{\alpha-n}=-\delta \text {, }
$$

where $\delta$ is Dirac's distribution. By Deny's theorem ${ }^{8)}$,

$$
D_{\alpha}=C_{\alpha, n} \text { pf. } r^{-\alpha-n}
$$

where

$$
C_{o, n}=\pi^{-n} \frac{I\left(\frac{n-\alpha}{2}\right) \Gamma\left(\frac{n+\alpha}{2}\right)}{\Gamma\left(\frac{\alpha}{2}\right) \Gamma\left(1-\frac{\alpha}{2}\right)}
$$

and the distribution pf. $r^{-\alpha-n}$ is defined as follows :

$$
\text { pf. } r^{-\alpha-n}(\varphi)=\text { pf. } \int|x|^{-\alpha-n} \varphi(x) d x^{9 !}
$$

for a function $\varphi$ of class $C^{\infty}$ with compact support.

Lemma 5. Let $f$ be a measurable function defined in $R^{n}$, and $x_{0}$ be a point in $R^{n}$. If $f$ is a function of class $C^{2}$ in a neighborhood of $x_{0}$ and

$$
\int_{\mathscr{C} B\left(x_{0} ; \varepsilon_{0}\right)}|y|^{-\alpha-n} f\left(x_{0}-y\right) d y<+\infty
$$

for a positive number $\varepsilon$, then $P_{f}^{\alpha}\left(x_{0}\right)$ exists and

$$
P_{f}^{\alpha}\left(x_{0}\right)=\text { pf. } \int|y|^{-\alpha-n} f\left(x_{0}-y\right) d y .
$$

Proof. Without loss of generality we may assume that $x_{0}=0 . \quad$ By our assumptions, for any $y$ in some neighborhood of 0 ,

$$
f(y)=f(0)+\sum_{i=1}^{n} y_{i} \frac{\partial f}{\partial y_{i}}(0)+\frac{1}{2} \sum_{i, j=1}^{n} y_{i} y_{j} \frac{\partial^{2} f}{\partial y_{i} \partial y_{j}}(0)+\psi(y),
$$

where $\psi(y)=o\left(\left|y^{2}\right|\right)$ and $y=\left(y_{1}, y_{2}, \ldots, y_{n}\right)$. Hence

$$
\int_{B(o ; \varepsilon)} \psi(y)|y|^{-\alpha-n} d y<+\infty
$$

for any sufficiently small positive number $\varepsilon$. Hence

8) Cf. [2], p. 153.

9) Ç. [9], p. 42 . 


$$
\text { pf. } \int f(-y)|y|^{-\alpha-n} d y
$$

exists, and

$$
\begin{aligned}
& \text { pf. } \int f(-y)|y|^{-\alpha-n} d y=\text { pf. } \int f(y)|y|^{-\alpha-n} d y \\
= & \lim _{\varepsilon \rightarrow 0}\left(\int_{\mathscr{C B}(o ; \varepsilon)}|y|^{-\alpha-n} f(y) d y+f(0) I^{(1)}(\varepsilon)+\sum_{i=1}^{n} \frac{\partial f}{\partial y_{i}}(0) I_{i}^{(2)}(\varepsilon)\right. \\
+ & \left.\frac{1}{2} \sum_{i, j=1}^{n} \frac{\partial^{2} f}{\partial y_{i} \partial y_{j}}(0) I_{i j}^{(3)}(\varepsilon)\right) .
\end{aligned}
$$

where $I^{(1)}, I_{i}^{(2)}$ and $I_{i j}^{(3)}$ are functions in $r(r=|y|)$ satisfying the following conditions :

$$
\begin{aligned}
& \frac{d I^{(1)}}{d r}(r)=\omega_{n} r^{-\alpha-1}, \\
& \frac{d I_{i}^{(2)}}{d r}(r)=r^{-\alpha-1} \int_{s_{1}} y_{i} d s, \\
& \frac{d I_{i j}^{(3)}}{d r}(r)=r^{-\alpha-1} \int_{s_{1}} y_{i} y_{j} d s,
\end{aligned}
$$

their integral constants are 0 ,

where $S_{1}$ is the unit sphere with center 0 and $d s$ is the area-element on $S_{1}$. Since $y_{i}$ and $y_{i} y_{j}(i \neq j)$ are harmonic in the usual sense in $R^{n}$,

$$
\int_{s_{1}} y_{i} d s=0 \text { and } \int_{s_{1}} y_{i} y_{j} d s=0(i \neq j) .
$$

On the other hand

$$
\int_{s_{1}} y_{i}^{2} d s=\frac{1}{n} \int_{s_{1}} y^{2} d s=\frac{\omega_{n}}{n}|y|^{2}
$$

Therefore

$$
\begin{aligned}
& \text { pf. } \int|y|^{-\alpha-n} f(y) d y \\
= & \lim _{\varepsilon \rightarrow 0}\left(\int_{\mathscr{C} B(0 ; \varepsilon)}|y|^{-\alpha-n} f(y) d y-\frac{\omega_{n}}{\alpha \varepsilon^{\alpha}} f(0)+\frac{\omega_{n}}{n(2-\alpha)} \varepsilon^{2-\alpha} \Delta f(0)\right) \\
= & \lim _{\varepsilon \rightarrow 0}\left(\int_{\mathscr{C} B(o ; \varepsilon)}|y|^{-\alpha-n} f(y) d y-\frac{\omega_{n}}{\alpha \varepsilon^{\alpha}} f(0)\right) \\
= & \lim _{\varepsilon \rightarrow 0} \frac{\omega_{n}}{\alpha \varepsilon^{\alpha}}\left(\mathscr{A}_{\alpha}(0 ; f, \varepsilon)-f(0)\right) .
\end{aligned}
$$

Consequently

$$
P_{f}^{\alpha}(0)=\text { pf. } \int|y|^{-\alpha-n} f(-y) d y
$$


This completes the proof.

\section{§ 2.6. Main theorems}

Theorem 1. Let $f$ be a Lebesgue measurable function defined in $R^{n}$ and $D$ be a domain in $R^{n}$. Assume that

(1) $f$ is lower semicontinuous and $f(x)>-\infty$ in $D$,

(2) $f$ is $\kappa_{x, r}$-integrable for any $x$ in $D$ and any open ball $B(x ; r)$ contained with its closure in $D$. Then $f$ is $\alpha$-superharmonic in $D$ if and only if $\underline{P}_{f}^{\alpha}(x) \leq 0$ in $D$.

Proof. First suppose that $f$ is $\alpha$-superharmonic in $D$. For any $x$ in $D$ and any open ball $B(x ; r)$ contained with its closure in $D$,

$$
\int_{\mathscr{C} B(x ; r)}|x-y|^{-\alpha-n}|f(y)| d y<+\infty
$$

In fact,

$$
\begin{aligned}
& \int_{\mathscr{C} B(x ; r)}|f(y)| \kappa_{x, r}(y) d y \\
= & a_{\alpha} r^{\alpha} \int_{\mathscr{C} B(x ; r)}|f(y)|\left(|y-x|^{2}-r^{2}\right)^{-\alpha / 2}|x-y|^{-n} d y \\
\geq & a_{\alpha} r^{\alpha} \int_{\mathscr{C} B(x ; r)}|f(y)||y-x|^{-\alpha-n} d y .
\end{aligned}
$$

Hence

$$
\int_{\mathscr{C} B(x ; r)}|x-y|^{-a-n}|f(y)| d y<+\infty .
$$

$f$ being $\alpha$-superharmonic in $D$, there exists a positive number $r_{x}$ such that

$$
f(x) \geq \mathfrak{M}_{\alpha}(x ; f, r)
$$

for any $0<r \leq r_{x}$. We take an arbitrary positive number $\varepsilon$ such that $\varepsilon<r_{x}$. Then

$$
\begin{aligned}
& \mathscr{A}_{\alpha}(x ; f, \varepsilon)-f(x) \\
= & \alpha \int_{1}^{\infty} \rho^{-\alpha-1}\left(\rho^{2}-1\right)^{\alpha / 2-1}\left(\mathfrak{R}_{\alpha}(x ; f, \varepsilon \rho)-f(x)\right) d \rho \\
\leq & \alpha \int_{r_{x / \varepsilon}}^{\infty} \rho^{-\alpha-1}\left(\rho^{2}-1\right)^{\alpha / 2-1}\left(\mathfrak{M}_{\alpha}(x ; f, \varepsilon \rho)-f(x)\right) d \rho .
\end{aligned}
$$

Now

$$
\alpha\left|\int_{\tau_{\not} / \varepsilon}^{\infty} \rho^{-\alpha-1}\left(\rho^{2}-1\right)^{\alpha / 2-1}\left(\mathbb{M}_{\alpha}(x ; f, \varepsilon \rho)-f(x)\right) d \rho\right|
$$




$$
\begin{aligned}
& \leq \alpha \int_{r_{x} / \varepsilon}^{\infty} \rho^{-\alpha-1}\left(\rho^{2}-1\right)^{\alpha / 2-1}\left|\mathfrak{M}_{\alpha}(x ; f, \varepsilon \rho)-f(x)\right| d \rho \\
& \leq \alpha \int_{r_{x} / \varepsilon}^{\infty} \rho^{-\alpha-1}\left(\rho^{2}-\left(\frac{r_{x}}{\varepsilon}\right)^{2}\right)^{\alpha / 2-1}\left|\mathfrak{M}_{\alpha}(x ; f, \varepsilon \rho)-f(x)\right| d \rho
\end{aligned}
$$

Putting $r=\frac{\varepsilon}{r_{x}} \rho$, we obtain

$$
\begin{aligned}
& \alpha \int_{r_{x} / \varepsilon}^{\infty} \rho^{-\alpha-1}\left(\rho^{2}-\left(\frac{r_{x}}{\varepsilon}\right)^{2}\right) \alpha / 2-1\left|\mathfrak{M}_{\alpha}(x ; f, \varepsilon \rho)-f(x)\right| d \rho \\
= & \alpha\left(\frac{\varepsilon}{r_{x}}\right)^{2} \int_{1}^{\infty} r^{-\alpha-1}\left(r^{2}-1\right)^{\alpha / 2-1}\left|\mathfrak{M}_{\alpha}\left(x ; f, r r_{x}\right)-f(x)\right| d r \\
\leq & \alpha\left(\frac{\varepsilon}{r_{x}}\right)^{2} \int_{1}^{\infty} r^{-\alpha-1}\left(r^{2}-1\right)^{\alpha / 2-1}\left(\mathfrak{M}_{\alpha}\left(x ;|f|, r r_{x}\right)+|f(x)|\right) d r \\
\leq & \left(\frac{\varepsilon}{r_{x}}\right)^{2}\left(\mathfrak{M}_{\alpha}\left(x ;|f|, r_{x}\right)+|f(x)|\right) .
\end{aligned}
$$

Since we may assume that $f(x)$ is finite, $\mathfrak{M}_{a}\left(x ;|f|, r_{x}\right)+|f(x)|$ is finite. Hence

$$
\underline{P}_{f}^{\alpha}(x) \leq \lim _{\varepsilon \rightarrow 0} \frac{\omega_{n} \varepsilon^{2-\alpha}}{\alpha r_{x}^{2}}-\left(\mathfrak{M}_{\alpha}\left(x ;|f|, r_{x}\right)+|f(x)|=0\right.
$$

In order to prove the converse, suppose that $\underline{P}_{f}^{\alpha}(x) \leq 0$ in $D$, and let $B\left(x_{0}\right.$; $r_{0}$ ) be an open ball contained with its closure in $D$. Then it is sufficient to prove the following inequality:

$$
f(x) \geq \int_{\mathscr{C} B\left(x_{0} ; r_{0}\right)} f(y) \lambda_{x_{0}, r_{0}}(x, y) d y
$$

in $B\left(x_{0} ; r_{0}\right)$. By the condition (2),

$$
\int|f(y)| \lambda_{x_{0}, r_{0}}(x, y) d y<+\infty .
$$

We take an open ball $B\left(x_{0} ; r_{1}\right)$ such that $\overline{B\left(x_{0} ; r_{0}\right)} \subset B\left(x_{0} ; r_{1}\right) \subset \overline{B\left(x_{0} ; r_{1}\right)} \subset D$. Since $f$ is lower semicontinuous and $f(x)>-\infty$ in $D$, there exists a sequence $\left(\varphi_{m}\right)$ of continuous functions with compact support in $R^{n}$ which tends increasing to $f$ on $\overline{B\left(x_{0} ; r_{1}\right)}$. Put

$$
f_{m}(x)=\left\{\begin{array}{l}
\varphi_{m}(x) \text { in } B\left(x_{0} ; r_{1}\right) \\
f(x) \text { on } \mathscr{C} B\left(x_{0} ; r_{1}\right) .
\end{array}\right.
$$

Then $\left(E_{f_{m}, B\left(x_{0} ; r_{0}\right)}\right)$ tends increasingly to $E_{f, B\left(x_{0} ; r_{0}\right)}$ as $m \rightarrow \infty$. Hence it is sufficient to prove that $f(x) \geq E_{f_{m}, B\left(x_{0} ; r_{0}\right)}(x)$ in $B\left(x_{0} ; r_{0}\right)$ for any $m$. Now let $\varphi$ be a function of class $C^{\infty}$ with compact support in $R^{n}$ such that $\varphi(x) \geq 0$ in $R^{n}$ and $\varphi(x)=1$ in $B\left(x_{0} ; r_{0}\right)$. And let $\mu_{\text {p }}$ be the balayaged measure of the 
measure $\varphi$ to $\mathscr{C} B\left(x_{0} ; r_{0}\right)$. Put

$$
g(x)=\int|x-y|^{\alpha-n} \varphi(y) d y-\int|x-y|^{\alpha-n} d \mu_{r}(y) .
$$

Then $g(x)$ is finite continuous in $R^{n}$ and $g(x)=0$ on $\mathscr{C} B\left(x_{0} ; r_{0}\right)$. Moreover for any $x$ in $B\left(x_{0} ; r_{0}\right), P_{g}^{\alpha}(x)$ exists and

$$
P_{g}^{\alpha}(x)=D_{\alpha} *\left(r^{\alpha-n} * \varphi\right)(x)-P_{U_{a}^{\alpha}}^{\mu_{2}}(x) .
$$

Since $S_{\mu_{\rho}}$ is contained in $\mathscr{C} B\left(x_{0} ; r_{0}\right) . \quad P_{U_{\alpha}^{\mu_{\varphi}}}^{\mu_{\varphi}}(x)=0$ in $B\left(x_{0} ; r_{0}\right)$. Hence

$$
D_{a} * g(x)=-\varphi(x)
$$

in $B\left(x_{0} ; r_{0}\right)$. Now for any positive number $\varepsilon$, we denote $E_{f_{m}, B\left(x_{0} ; r_{0}\right)}-f-\varepsilon g$ by $h$. The function $h$ is upper semicontinuous and $h(x)<+\infty$ in $B\left(x_{0} ; r_{1}\right)$, and it is equal to 0 on $\mathscr{C} B\left(x_{0} ; r_{1}\right)$. By Lemma $2, E_{f_{m}, B\left(x_{0} ; r_{0}\right)}$ is $\alpha$-harmonic in $B\left(x_{0} ; r_{0}\right)$. Suppose that there exists a point $x_{1}$ in $B\left(x_{0} ; r_{0}\right)$ such that $h\left(x_{1}\right)>0$ and

$$
h\left(x_{1}\right)=\sup \left\{h(x) ; x \in B\left(x_{0} ; r_{0}\right)\right\} .
$$

Then for any open ball $B\left(x_{1} ; r\right)$ contained with its closure in $B\left(x_{0} ; r_{0}\right)$,

$$
\begin{aligned}
& \mathscr{A}_{\alpha}\left(x_{1} ; h, r\right)=\frac{\alpha r^{\alpha}}{\omega_{n}} \int_{\mathscr{C} B\left(x_{1} ; r_{0}\right)}\left|x_{1}-y\right|^{-\alpha-n} h(y) d y \\
\leq & \frac{\alpha r^{\alpha}}{\omega_{n}} \int_{\mathscr{C}\left(x_{1} ; r\right) \cap B\left(x_{0} ; r_{0}\right)}\left|x_{1}-y\right|^{-\alpha-n} h(y) d y \\
\leq & \frac{\alpha r^{\alpha}}{\omega_{n}} \int_{\mathscr{C}\left(x_{1} ; r\right) \cap B\left(x_{0} ; r_{0}\right)}\left|x_{1}-y\right|^{-\alpha-n} h\left(x_{1}\right) d y \\
< & \frac{\alpha r^{\alpha}}{\omega_{n}} \int_{\mathscr{C} B\left(x_{1} ; r\right)}\left|x_{1}-y\right|^{-\alpha-n} h\left(x_{1}\right) d y=h\left(x_{1}\right) .
\end{aligned}
$$

Hence

$$
\varlimsup_{\varepsilon \rightarrow 0} \frac{\omega_{n}}{\alpha \varepsilon^{\alpha}}\left(\mathscr{A}_{\alpha}\left(x_{1} ; h, \varepsilon\right)-h\left(x_{1}\right)\right) \leq 0 .
$$

On the other hand

$$
\underline{P}_{-h}^{\alpha}(x) \leq-\varepsilon \varphi(x)=-\varepsilon
$$

in $B\left(x_{0} ; r_{0}\right)$. This is a contradiction. Consequently $h(x) \leq 0$ in $B\left(x_{0} ; r_{0}\right)$, i.e.,

$$
E_{f_{m}, B\left(x_{0} ; r_{0}\right)}(x) \leq f(x)
$$

in $B\left(x_{0} ; r_{0}\right)$. Therefore

$$
f(x) \geq E_{f, R\left(x_{0} ; r_{0}\right)}(x)
$$


in $B\left(x_{0} ; r_{0}\right)$. In particular

$$
f(x) \geq \mathbb{M}_{\alpha}\left(x_{0} ; f, r\right),
$$

i.e., $f$ is $\alpha$-superharmonic in $D$. This completes the proof.

Theorem 2. Let $D$ be a domain in $R^{n}$ and a function $f$ defined in $R^{n}$ be finite continuous in $D$. Then $f$ is $\alpha$-harmonic in $D$ if and only if $P_{f}^{\alpha}(x)$ exists in $D$ and $P_{f}^{\alpha}(x)=0$ in $D$.

Proof. Suppose that $P_{f}^{\alpha}(x)=0$ in $D$. Since

$$
\int_{\mathscr{C} B(x ; r)}|x-y|^{-\alpha-n}|f(y)| d y<+\infty
$$

for any in $D$ and any positive number $r$, it holds that

$$
\int_{\mathscr{C} B(x ; r)}|f(y)| \kappa_{x, r}(y) d y<+\infty
$$

for any $x$ in $D$ and any open ball $B\left(x_{0} ; r\right)$ contained with its closure in $D$. Consequently, by Theorem $1, f$ is $\alpha$-harmonic in $D$. The converse is evident by Theorem 1 .

\section{Chapter 3. Ninomiya's dominarion principle}

In this chapter, we assume that $0<\alpha \leq 2,0<\alpha<2$ or $0<\alpha<1$ according to $n \geq 3, n=2$ or $n=1$.

TheOREм $3 .^{10)}$ Let $\mu$ be a positive measure with compact support such ihat

$$
\iint|x-y|^{\alpha-n} d \mu(y) d \mu(x)<+\infty,
$$

and let $\nu$ be a positive measure. If

$$
U_{\alpha}^{\mu}(x) \leq U_{\alpha}^{\nu}(x)
$$

on $S_{\mu}$, then

$$
U_{\beta}^{\mu}(x) \leq U_{\beta}^{\nu}(x)
$$

in $R^{n}$ for any $\beta$ such that $\alpha \leq \beta<n$.

Proof. By Ninomiya's theorem ${ }^{11}$, it is sufficient to prove the following

10) N. Ninomiya [7] proved this when $n \geq 3$. An alternate proof of this theorem was given in [5].

11) Cf. [6], p. 142 . 
assertion. Let $\alpha$ and $\beta$ be the same as Theorem 3 , let $\lambda$ be a positive measure with compact support, and let $p$ be a point in $\mathscr{C} S_{\lambda}$. If

$$
U_{a}^{\lambda}(x) \leq|x-p|^{\beta-n}
$$

in $S_{\lambda}$, then

$$
U_{\alpha}^{\lambda}(x) \leq|x-p|^{\beta-n}
$$

in $R^{n}$. To exclude the trivial case, we may assume that $\alpha<\beta$. First we shall show that $|x-p|^{\beta-n}$ is $\alpha$-superharmonic in $R^{n}$. In fact, by M. Riesz's formula ${ }^{12)}$,

$$
|x-p|^{\beta-n}=\frac{1}{K_{\alpha, \beta-\alpha}} \int|x-y|^{\alpha-n}|y-p|^{(\beta-\alpha)-n} d y,
$$

where

$$
K_{x, \beta-\alpha}=\pi^{n / 2} \frac{\Gamma\left(\frac{\alpha}{2}\right) \Gamma\left(\frac{\beta-\alpha}{2}\right) \Gamma\left(\frac{n-\beta}{2}\right)}{\Gamma\left(\frac{n-\alpha}{2}\right) \Gamma\left(\frac{n-\beta+\alpha}{2}\right) \Gamma\left(\frac{\beta}{2}\right)} .
$$

Since the measure $\frac{1}{K_{a, \beta-\alpha}}|y-p|^{(\beta-\alpha)-n}$ is an $\alpha$-finite positive measure, $\mid x-$ $\left.p\right|^{\beta-n}$ is $\alpha$-superharmonic in $R^{n}$. On the other hand, $U_{\alpha}^{\lambda}(x)$ is $\alpha$-harmonic in $\mathscr{C} S_{\lambda}$. Put

$$
f(x)=|x-p|^{\beta-n}-U_{\alpha}^{\lambda}(x) .
$$

Then $f$ is $\alpha$-superharmonic in $\mathscr{C} S_{\lambda}$. Next we shall show that $f$ is non-negative at infinity. In fact, let $\varepsilon$ be a positive number. Then $S_{\lambda}$ being compact, there exists a positive number $\rho$ such that

$$
|x-y|^{\alpha-n} \leq(1+\varepsilon)|x-p|^{\alpha-n}
$$

for any $x$ in $\mathscr{C} B(O ; \rho)$ and any $y$ in $S_{\lambda}$. Hence for any $x$ in $\mathscr{C} B(O ; \rho)$,

$$
U_{\alpha}^{\lambda}(x) \leq(1+\varepsilon) \lambda\left(R^{n}\right)|x-p|^{\alpha-n} .
$$

Since $\beta>\alpha$, there exists a positive number $R_{0}$ such that $R_{0} \geq \rho, S_{\lambda}$ is contained in $B\left(O ; R_{0}\right)$ and

$$
|x-p|^{\beta-n} \geq(1+\varepsilon) \lambda\left(R^{n}\right)|x-p|^{\alpha-n}
$$

for any in $\mathscr{C} B\left(O ; R_{0}\right)$. Finally put

12) Cf. [2], p. 151. 


$$
\bar{f}(x)=\left\{\begin{array}{l}
f(x) \text { in } \mathscr{Q} S_{\lambda}, \\
\lim _{\substack{n \rightarrow x \\
y \in \mathscr{C} S}} f(y) \text { on the boundary of } \mathscr{C} S_{\lambda} .
\end{array}\right.
$$

Then $\bar{f}$ is lower semicontinuous on $\overline{\mathscr{C} S} S_{\lambda}$ and $\bar{f}$ is non-negative at infinity. By Frostman's theorem ${ }^{13)}$,

$$
\bar{f}(x) \geq 0
$$

on $\partial \mathscr{C} S_{\lambda}$. Hence there exists $x_{1}$ in $\left.\overline{\mathscr{C} S_{\lambda}} \cap \overline{B(O} ; R_{0}\right)$ such that $\bar{f}\left(x_{1}\right)$ attains the minimum of $\bar{f}(x)$ on $\left.\overline{\mathscr{C} S_{\lambda}} \cap \overline{B\left(O ; R_{0}\right.}\right)$. Assume that $\bar{f}\left(x_{1}\right)$ is negative. Then $x_{1}$ is contained in $\mathscr{C} S_{\lambda}$. For any ball $B\left(x_{1} ; r\right)$ contained with its closure in $\mathscr{C} S_{\curlywedge}$,

$$
\begin{aligned}
& \mathfrak{M}_{\alpha}\left(x_{1} ; f, r\right)=\int f(y) \kappa_{x_{1}, r}(y) d y \\
\geq & \int_{\left.\mathscr{C} S_{\lambda} \cap B \backslash o ; R_{0}\right)} f(y)_{\kappa_{x_{1}, r}}(y) d y \geq \int_{\mathscr{C} \aleph_{\lambda} \cap B\left(o ; R_{0}\right)} f\left(x_{1}\right) \kappa_{x_{i}, r}(y) d y \\
> & \int f\left(x_{1}\right) \kappa_{x_{1}, r}(y) d y=f\left(x_{1}\right) .
\end{aligned}
$$

This contradicts the $\alpha$-superharmonicity of $f$. Consequently

$$
U_{\alpha}^{\lambda}(x) \leq|x-p|^{\beta-n}
$$

in $R^{n}$. This completes the proof.

\section{REFERENCES}

[1] M. Brelot: Éléments de la théorie classique du potentiel, Les cours de Sorbonne, 3e cycle, Centre de Documentation Universitaire, Paris, 1960.

[2] J. Deny: Les potentiels d'énergie finie, Acta Math., 82 (1950), 107-183.

[3] O. Frostman: Potentiel d'équilibre et capacité des ensembles, Comm. Sem. Math., Lund, 3 (1935), 1-118.

[4] O. Frostman: Sur les fonctions surharmonique d'ordre fractionnaire, Ark. Math. Astv. Fysik., 16 (1939), 16-35.

[5] M. Itó: Remarks on Ninomiya's domination principle, Proc. Jap. Acad., 40 (1964), 743746.

[6] N. Ninomiya: Sur un principe du maximum dans la théorie du potentiel, Jour. Math., Osaka City Univ., 12 (1961), 139-143.

[7] N. Ninomiya: Sur un principe du maximum pour la potentiel du Riesz-Frostman, Jour. Math., Osaka City Univ., 13 (1962), 57-62.

[8] M. Riesz: Intégrales de Riemann-Liouville et potentiels, Acta Sci. Math., Szeged, 9 (1938), 1-42.

[9] L. Schwartz: Théorie des distributions 1, Paris Hermann, 1951.

\section{Mathematical Institute}

Nagoya University

13) Cf. $[3]$, p. 69 . 